\title{
FLUJO SALIVAL EN ADULTOS MAYORES Y SU RELACIÓN CON ENFERMEDADES CRÓNICAS Y EL CONSUMO DE MEDICAMENTOS *
}

\author{
${ }^{1}$ Silvia Marcela López, ${ }^{1}$ Luis Francisco Ochoa, ${ }^{2}$ Nhora Milena Valle, ${ }^{3}$ Gloria Cristina Aránzazu M. \\ ${ }^{1}$ Estudiante IX semestre, F. de Odontología, U. Santo Tomas, ${ }^{2}$ Estudiante X semestre, \\ F. de Odontología, U. Santo Tomas, ${ }^{3}$ Odontóloga U. Santo Tomás, Especialista en Patología y \\ Medios Diagnósticos U. El Bosque, Docente U. Santo Tomas
}

Autor responsable de correspondencia: Gloria Cristina Aránzazu Moya

Dirección de correo electrónico: aranzazugloria@yahoo.es

Mejor trabajo de investigación en el Área de Epidemiología. XVI Encuentro Nacional de Investigación Odontológica ACFO,
Cartagena - septiembre de 2005

\section{RESUMEN}

Objetivo: Determinar la cantidad de flujo salival en pacientes que presentan compromisos sistémicos y/o un consumo crónico de medicamentos y que asisten a las clínicas odontológicas de la Universidad Santo Tomás.

Materiales y Métodos: Se desarrolló un estudio observacional analítico de corte transversal en una población de 110 sujetos, con edades entre los 50 y los 85 años. Se tuvieron en cuenta variables sociodemográficas y las relacionadas con el consumo de medicamentos, tipo de medicamentos y condición sistémica. Se usó el test Chi cuadrado y test exacto de Fisher para variables cualitativas, para variables cuantitativa t Student y/o Test de Rangos de Wilcoxon.

Resultados: De los pacientes con compromiso sistémico se presentaron con mayor frecuencia los que padecían hipertensión 37 (33.6\%) de los cuales presentaron NFSE anormal 27 (60\%) y NFSR anormal 30 (53.5\%). Los que ingerían medicamentos de tipo antihipertensivo 39 (35.4\%) presentaron anormalidad en los NFSE 27 (60\%), y además presentaron una disminución de sus NFSR 30 (53.57\%). Por grupo de condición sistémica y consumo de medicamentos se observaron diferencias estadísticamente significativas en cuanto a flujo salival estimulado y a flujo salival en reposo, se refiere $(\mathrm{p}<0.001)$. La frecuencia de la práctica deportiva reportó una relación protectora de la disminución de flujo salival de 0.63 I.C. [0.41;0.97] ( $\mathrm{p}=0.04$ ). Igualmente, sucedió con la variable de consumo de alimentos sólidos en el cual se encontró una relación protectora 0.61 I.C. [0.37; 1.02] $(\mathrm{p}=0.063)$.

Conclusiones: Se pudo determinar que existe una relación directa entre el consumo de medicamentos y la disminución de los niveles de flujo salival estimulado y en reposo. [López SM, Ochoa LF, Valle NM, Aránzazu GC. Flujo salival en adultos mayores y su relación con enfermedades crónicas y el consumo de medicamentos. Revista Ustasalud Odontología 2007; 6: 9 - 16]

Palabras clave: Xerostomía, Hiposalivación, Hipertensión, Fármacos antihipertensivos.

\section{SALIVARY FLOW IN THE ELDERLY AND THEIR RELATION WITH CHRONIC DISEASES AND MEDICINE CONSUMPTION}

\begin{abstract}
Objective: To determine salivary flow rates in patients that have a systemic condition and consumption of medicines who attend the dental clinics of Santo Tomas University.

Materials and Methods: An analytic study of transverse court was done in a population of 110 elderly people, with ages among the 50 and the 85 years. For the study they were kept in mind socio-demographic variables and those related with the consumption of medications, type of medications, systemic condition. These variables were evaluated by means of statistical tests as: test squared Chi and exact test of Fisher, for variables of quantitative type $t$ student and/or Mann Whithey.

Results: Of the patients with systemic commitment those appeared most frequently that suffered hypertension 37 (33,6\%) of which showed abnormal NFSE 27 (60\%) and abnormal NFSR 30 (53.5). Those that 39 ingested medicines of the antihypertensive type (35,4\%) showed abnormality in NFSE 27 (60\%), and in addition displayed a reduction of their NFSR 30 (53,57\%). By group of systemic condition and medicine consumption statistically significant differences as far as stimulated salivary flow were observed and to salivary flow in rest, it was about $(p<0,001)$. The frequency of the sport practice reported a protective relation of the diminution of salivary flow of 0,63 I.C. $[0.41 ; 0.97](p=0.04)$. Also, it happened to the variable of solid food consumption in which was a protective relation 0,61 I.C. $[0.37 ; 1.02](\mathrm{p}=0.063)$.

Conclusions: It was possible to be determined that a direct relation between the medicine consumption and the diminution of the levels of stimulated salivary flow exists and in rest.
\end{abstract}

Key words: Xerostomia, Hyposalivation, Hypertension, Antihypertensive drugs.

Recibido para publicación: 12 de abril de 2007. Aceptado para publicación: 22 de julio de 2007.

* Grupo de Investigación Salud Integral y Bienestar del Adulto Mayor (SIBAM) 


\section{INTRODUCCIÓN}

La disminución del flujo salival en el adulto mayor es una realidad y un gran problema para el paciente y para el odontólogo por los efectos deletéreos que tienen sobre varias estructuras buco dentales, esta disminución del flujo salival produce una patología conocida como xerostomía o síndrome de boca seca la cual va a complicar el estado de la cavidad oral de dichos pacientes. ${ }^{1}$

La saliva es una secreción exocrina compleja dada por las glándulas salivales mayores y menores, importante en el mantenimiento de la homeostasis de la cavidad bucal. ${ }^{2,3}$ Sus funciones en relación con el flujo y la composición molecular (proteínas, glucoproteínas y fosfoproteínas), son proteger los tejidos bucales contra la desecación y las agresiones del medio ambiente, armonizar los procesos de desmineralización, remineralización, actividad antimicrobiana y lubricación de las superficies oclusales y mantener el balance ecológico que inhibe la adherencia y desarrollo bacteriano por medio de la IgA, 2,4,5 así como también nivela el PH y ayuda a mantener la integridad de los tejidos dentales. ${ }^{2,6}$

La disminución del flujo salival, que en ocasiones puede ser producida por la ingesta crónica de medicamentos, trae como consecuencias diferentes patologías como: caries cervical, xerostomía, daño de las glándulas salivales mayores y menores, ${ }^{2}$ y problemas en el manejo y adaptación de las prótesis dentales.

La xerostomía se define como el síntoma o sensación subjetiva de boca seca; clínicamente, aparece como sequedad, palidez y atrofia de las mucosas y sus síntomas son dolor, ardor, sensación de quemazón, inflamación de la lengua, dificultad para deglutir y dificultad para masticar, entre otros. ${ }^{7-10}$ Se presenta con relativa frecuencia en personas adultas, ${ }^{8}$ aunque no es específica de esa edad; predomina en pacientes que superan la quinta edad de la vida, ${ }^{7}$ en los adultos mayores su prevalencia varía de un 13 a $28 \%$ en la población adulta, y aumenta a un $60 \%$ en la población mayor de 60 años. Se ha observado una relación estrecha entre los medicamentos antihipertensivos (diuréticos, los beta bloqueadores, y los inhibidores de la enzima convertidora de angiotensina) con la aparición de xerostomía. ${ }^{8,11}$

Múltiples estudios han podido determinar que la ingesta crónica de medicamentos puede generar diversas alteraciones en la función de las glándulas salivales y, por tanto, producir disminución del flujo salival. Sin embargo, también existen estudios que se oponen a estas afirmaciones, por lo cual hay aún controversia.
El propósito de este estudio fue determinar la cantidad de flujo salival en pacientes que presentan compromisos sistémicos y/o que presenten un consumo crónico de medicamentos y que asisten a las clínicas odontológicas de la Universidad Santo Tomás.

\section{MATERIALES Y MÉTODOS}

Se realizó un estudio observacional analítico de corte transversal. La población estuvo constituida por los pacientes de ambos géneros que asistían a consulta odontológica a las clínicas de la Universidad Santo Tomás. Los criterios de inclusión definidos para el estudio fueron: pacientes mayores de 50 años que asistían a consulta a las clínicas odontológicas de la USTA, sometidos o no a medicación crónica para control de enfermedades sistémicas.

El tamaño de la muestra se determinó mediante la rutina sample size del paquete estadístico EPI INFO 6.0 , se consideró un nivel de confianza del $95 \%$ con un poder del $80 \%$ y relación de expuesto no expuesto 1 a 1 y OR de 3.5, una frecuencia de xerostomía en pacientes bajo medicación del $70 \%$ y del $40 \%$ en aquellos no expuestos a medicamentos con un RR 1,75 OR 3.5 por lo cual una muestra de 96 pacientes permitiría obtener diferencias estadísticamente significativas, La muestra se seleccionó mediante un muestreo probabilístico de tipo aleatorio simple.

A cada paciente se le recolectaron los datos por medio de una encuesta, en la cual se tuvieron en cuenta variables sociodemográficas (edad y género), condición sistémica, medicamentos utilizados, subjetivas de xerostomía, práctica de deporte, vasos de agua que consume al día, tipo de dieta, disminución de la masticación, presencia de dentadura artificial, nivel de flujo salival estimulado y en reposo y alteración de la función oral.

Previa a la recolección de los datos se efectuó una prueba piloto, en la que se verificaron instrumentos y la comprensión de las preguntas, se estandarizaron los procesos y los tiempos requeridos para responder la encuesta y efectuar los procedimientos.

Se tomó la muestra de flujo salival de acuerdo al siguiente procedimiento para recolección de flujo salival estimulado: se solicitó al paciente que no consumiera alimentos 1 hora antes de la recolección de saliva, al momento de la toma se le solicitó que se enjuagara la boca, se le suministraron 2 gramos de cera marca WHIP MIX y se le pidió que la mordiera durante 1 minuto, se retiró la cera y se le solicitó que depositara la saliva en un tubo milimetrado durante 4 minutos, luego se hizo la lectura y se recolectó la información en el formato correspondiente. Para la recolección del flujo salival en reposo se llevaron a cabo los siguientes pasos: se le pidió al paciente 
que descansara un poco, y que luego depositara la saliva contenida en ese momento en su boca dentro del tubo milimetrado, posteriormente se hizo la lectura y se recopiló la información en el formato correspondiente. ${ }^{1}$

La base de datos se realizó en Excel por duplicado. Se exportó al paquete estadístico EPI-INFO 6.0 y mediante la rutina validate se verificó la calidad de la digitación. El análisis final se realizó en STATA 8.0.

Para el análisis univariado, se tuvieron en cuenta para variables cuantitativas medidas de resumen de tendencia central y dispersión y para variables cualitativas las variables se resumieron en proporciones y se presentaron en tablas de frecuencia. Para el análisis bivariado se tuvo en cuenta para variables cualitativas test de Chi cuadrado y test exacto de Fisher; para las variables cuantitativas $t$ student y test de rangos de Wilcoxon según la distribución de la variable.

Para el análisis multivariado, se aplicó un análisis de regresión binomial que estimó los RR (Riesgos Relativos:Razón de Prevalencia) y sus respectivos intervalos de confianza del $95 \%$. Se definió como variables de salidas nivel de flujo salival estimulado y en reposo y como variables explicatorias a las variables sociodemográficas, medicación y condición sistémica, práctica deportiva y aspectos re- lacionados con la hidratación. Cada una de las variables explicatorias se relacionó mediante análisis de regresión binomial bivariado y a partir de éste, se seleccionaron para el modelo final las variables que obtuvieron una probabilidad igual o menor a $\mathrm{p}=0.25$ y aquellas que se consideraron biológicamente relacionadas. Para el análisis se consideró un nivel de significancia de Alfa $(\alpha)=0.05$.

El presente estudio se fundamentó en las recomendaciones éticas establecidas en la resolución 008430 de 1993 del Ministerio de Protección Social de la República de Colombia, que según el título 11 en su artículo 11, clasificó a la presente investigación como de riesgo mínimo. Recibió la aprobación del Comité de Ética institucional, previa recolección de la información. Se garantizó la privacidad y la confidencialidad y autonomía en el manejo de la información recopilada en cada una de las personas que hicieron parte del estudio. Decidieron participar libremente en el estudio después de firmar la carta de consentimiento informado.

\section{RESULTADOS}

Se encuestaron 110 personas con una media de edad $65.8 \pm 10.27$ años y una mediana de 63.55 años con rangos entre 50 y 85 años. El grupo de edad que más se presentó fue de 50 a 60 años 43 (39.1\%) (Tabla1).

Tabla 1. Descripción de la población de adultos mayores según las variables sociodemográficas considerado en forma global, según N.F.S.E. y N.F.S.R.

\begin{tabular}{|c|c|c|c|c|c|}
\hline \multirow[t]{2}{*}{ VARIABLES } & \multirow{2}{*}{$\begin{array}{l}\text { GLOBAL } \\
\mathrm{n}=110 \\
\text { Frec. (\%) }\end{array}$} & \multirow{2}{*}{$\begin{array}{c}\text { N.F.S.E. } \\
\text { ANORMAL } \\
\mathrm{n}=45 \\
\text { Frec. (\%) }\end{array}$} & \multirow[t]{2}{*}{$\mathbf{P}^{*}$} & $\begin{array}{c}\text { N.F.S.R. } \\
\text { ANORMAL } \\
\text { n=56 }\end{array}$ & \multirow[t]{2}{*}{$\mathbf{P}^{*}$} \\
\hline & & & & Frec. (\%) & \\
\hline \multicolumn{6}{|l|}{ EDAD } \\
\hline $50-60$ & $43(39,1)$ & $19(42,2)$ & 0,494 & $16(28,5)$ & 0,130 \\
\hline $61-70$ & $30(27,3)$ & $13(28,9)$ & & $17(30,36)$ & \\
\hline $71-80$ & $25(22,7)$ & $7(15,6)$ & & $15(26,8$ & \\
\hline $81-90$ & $12(10,9)$ & $6(13,3)$ & & $8(14,3)$ & \\
\hline \multicolumn{6}{|l|}{ GÉNERO } \\
\hline Mujeres & $60(54,6)$ & $26(57,8)$ & 0,571 & $33(58,9)$ & 0,347 \\
\hline Hombres & $50(45,4)$ & $19(42,2)$ & & $23(41,07)$ & \\
\hline \multicolumn{2}{|l|}{ * Test Chi cuadrado } & & $\alpha=0$ & & \\
\hline \multicolumn{2}{|c|}{$\begin{array}{l}\text { De los pacientes con compromiso sistémico se pre- } \\
\text { sentaron en mayor cantidad los que padecían de } \\
\text { hipertensión } 37(33.6 \%) \text { de los cuales presentaron } \\
\text { N.F.S.E. anormal } 27(60 \%) \text { y N.F.S.R. anormal } 30 \\
\text { (53.5). De los que tomaban medicamentos los que } \\
\text { ingerían los de tipo antihipertensivos } 39(35.4 \%) \\
\text { presentaron anormalidad en los N.F.S.E. } 27(60 \%) \\
\text { y además presentaron una disminución de sus }\end{array}$} & \multicolumn{4}{|c|}{$\begin{array}{l}\text { N.F.S.R. } 30(53.57 \%) \text {. Por grupo de condición sisté- } \\
\text { mica y consumo de medicamentos se observaron di- } \\
\text { ferencias estadísticamente significativas en cuanto } \\
\text { a flujo salival estimulado y a flujo salival en reposo } \\
\text { se refiere }(p<0.001)(\text { Tabla } 2) \text {. Con relación a la prác- } \\
\text { tica de deporte en cuanto a los N.F.S.E. anormales } \\
25(55.6 \%) \text { se observaron diferencias marginalmen- } \\
\text { te significativas }(p=0.064) \text { y para los N.F.S.R. anor- }\end{array}$} \\
\hline
\end{tabular}


males 32 (57.1\%) se encontraron diferencias estadísticamente significativa $(p=0.037)$. En cuanto al consumo de vasos de agua por día se reportó una media de $4.5 \pm 1.9$ de vasos y una mediana de 4 vasos y un rango de 1 a 10 (Tabla 3). La alimentación que predominó en la población fue de tipo blanda 61 (55.45\%); donde se encontró una anormalidad de los N.F.S.R. 38 (67.86\%), observándo- se una diferencia estadísticamente significativa $(\mathrm{p}=0.016)$, además se presentó disminución de la masticación en $24(21.82 \%)$ a los cuales se les encontró una anormalidad en los N.F.S.E. 16 (35.6\%) con una diferencia estadísticamente significativa $(\mathrm{p}=0.004)$ y anormalidad de los N.F.S.R. 19 (33.9\%) con una diferencia estadísticamente significativa $(\mathrm{p}=0.002)$ (Tabla 4).

Tabla 2. Descripción de la población adulta mayor según la condición sistémica y consumo de medicamentos relacionado en forma global, según N.F.S.E. y N.F.S.R.

\begin{tabular}{|c|c|c|c|c|c|}
\hline \multirow[t]{2}{*}{ VARIABLES } & \multirow{2}{*}{$\begin{array}{l}\text { GLOBAL } \\
\mathrm{n}=110 \\
\text { Frec. (\%) }\end{array}$} & \multirow{2}{*}{$\begin{array}{c}\text { N.F.S.E. } \\
\text { ANORMAL } \\
\mathrm{n}=45 \\
\text { Frec. (\%) }\end{array}$} & \multirow[t]{2}{*}{$\mathbf{P}+$} & $\begin{array}{c}\text { N.F.S.R. } \\
\text { ANORMAL } \\
\text { n=56 }\end{array}$ & \multirow[t]{2}{*}{$\mathbf{P +}$} \\
\hline & & & & Frec. (\%) & \\
\hline \multicolumn{6}{|l|}{ ENFERMEDADES } \\
\hline Ninguna & $50(45,4)$ & $11(24,4)$ & $<0,001^{* *}$ & $18(32,14)$ & $<0,001^{* *}$ \\
\hline Ansiedad & $12(10)$ & $5(11,1)$ & & $5(8,93)$ & \\
\hline Depresión & $2(1,8)$ & --- & & $1(1,8)$ & \\
\hline Anemia & $1(0,9)$ & ---- & & ---- & \\
\hline Hipertensión & $37(33,6)$ & $27(60)$ & & $30(53,5)$ & \\
\hline Otros & $9(8,2)$ & $2(4,4)$ & & $2(3,57)$ & \\
\hline \multicolumn{6}{|c|}{ CONSUMO DE MEDICAMENTO } \\
\hline $\mathrm{Si}$ & $54(49,1)$ & $32(71,1)$ & $<0,001^{*}$ & $37(66,07)$ & $<0,001^{*}$ \\
\hline No & $56(50,9)$ & $13(28,9)$ & & $19(33,9)$ & \\
\hline \multicolumn{6}{|l|}{ TIPO DE MEDICAMENTO } \\
\hline Ninguno & $56(50,9)$ & $13(28,9)$ & $<0,001^{* *}$ & $19(33,9)$ & $<0,001^{* *}$ \\
\hline Antihipertensivo & $39(35,4)$ & $27(60)$ & & $30(53,57)$ & \\
\hline Ansiolítico & $12(10,9)$ & $4(8,9)$ & & $6(10,71)$ & \\
\hline Antiglicémico & $1(0,9)$ & $1(2,2)$ & & $1(1,8)$ & \\
\hline Anticonvulsionantes & $1(0,9)$ & ---- & & ---- & \\
\hline Broncodilatadores & $1(0,9)$ & ---- & & ---- & \\
\hline
\end{tabular}

Tabla 3. Sudoración excesiva y práctica deportiva relacionado con los niveles de flujo salival estimulado y en reposo disminuidos.

\begin{tabular}{|c|c|c|c|c|c|}
\hline \multirow[t]{2}{*}{ VARIABLES } & \multirow{2}{*}{$\begin{array}{l}\text { GLOBAL } \\
\mathrm{n}=110 \\
\text { Frec. }(\%)\end{array}$} & \multirow{2}{*}{$\begin{array}{c}\text { N.F.S.E. } \\
\text { ANORMAL } \\
\mathrm{n}=45 \\
\text { Frec. (\%) }\end{array}$} & \multirow[t]{2}{*}{$\mathbf{P}$} & $\begin{array}{c}\text { N.F.S.R. } \\
\text { ANORMAL } \\
\text { n=56 }\end{array}$ & \multirow[t]{2}{*}{$\mathbf{P}$} \\
\hline & & & & Frec. (\%) & \\
\hline \multicolumn{6}{|l|}{ SUDORACIÓN EXCESIVA } \\
\hline Fiebre & $1(0,9)$ & $1(2,2)$ & \multirow[t]{3}{*}{$0,50^{* *}++$} & $1(1,8)$ & \multirow[t]{3}{*}{$0,670^{* *}$} \\
\hline Sudor abundante & $30(27,2)$ & $11(24,4)$ & & $14(25)$ & \\
\hline No hubo sudoración & $79(71,8)$ & $33(73,33)$ & & $41(73,21)$ & \\
\hline \multicolumn{6}{|c|}{ FREC. DE PRACTICA DEPORTIVA } \\
\hline $\mathrm{Si}$ & $73(66,4)$ & $25(55,6)$ & \multirow[t]{2}{*}{$0,064^{* *}++$} & $32(57.1)$ & \multirow[t]{2}{*}{$0.037^{*}+$} \\
\hline No & $37(33,6)$ & $20(44,4)$ & & 24 (42.9) & \\
\hline * Test Chi cuadrado & Test exacto de & & & $x=0.05$ & \\
\hline
\end{tabular}


Tabla 4. Signos y síntomas y su relación con el flujo salival estimulado y en reposo disminuidos.

\begin{tabular}{|c|c|c|c|c|c|}
\hline \multirow[t]{2}{*}{ VARIABLES } & $\begin{array}{c}\text { GLOBAL } \\
\mathrm{n}=110\end{array}$ & $\begin{array}{c}\text { N.F.S.E. } \\
\text { ANORMAL } \\
\text { n=45 }\end{array}$ & $\mathbf{P}$ & $\begin{array}{c}\text { N.F.S.R. } \\
\text { ANORMAL } \\
\text { n=56 }\end{array}$ & \multirow[t]{2}{*}{$P$} \\
\hline & Frec. (\%) & Frec. (\%) & & Frec. (\%) & \\
\hline \multicolumn{6}{|l|}{ SIGNOS Y SÍNTOMAS } \\
\hline Ardor Bucal & $16(14,5)$ & $9(20)$ & $0,177^{*}$ & $11(19,64)$ & $0,123^{*}$ \\
\hline Dificultad masticatoria & $11(10)$ & $5(11,1)$ & $0,747^{*}$ & $7(12,5)$ & $0,373^{*}$ \\
\hline Dificultad para hablar & $10(9,1)$ & $6(13,3)$ & $0,312^{* *}$ & $8(14,3)$ & $0,094^{* *}$ \\
\hline frecuencia de boca seca & $22(20)$ & $13(28,9)$ & $0,052^{* *}++$ & $16(28,5)$ & $0,022^{*}+$ \\
\hline Mal aliento & $26(23,6)$ & $13(28,9)$ & $0,281^{*}$ & $16(28,5)$ & $0,215^{*}$ \\
\hline Disminución masticación & $24(21,8)$ & $16(35,6)$ & $0,004^{*}+$ & $19(33,9)$ & $0,002^{*}+$ \\
\hline Consumo de alimentos blandos & $61(55,4)$ & $30(66,7)$ & $0,143^{* *}$ & $38(67,8)$ & $0,016^{* *}+$ \\
\hline Consumo de alimentos sólidos & $46(41,8)$ & $14(31,1)$ & & $17(30,4)$ & \\
\hline Consumo de alimentos líquidos & $3(2,73)$ & $1(2,2)$ & & $1(1,8)$ & \\
\hline${ }^{*}$ Test Chi cuadrado & ${ }^{* *}$ Test exacto de & & $\alpha=0.05$ & & \\
\hline
\end{tabular}

En relación con las prótesis dentales el 53(30\%) reportó problemas con el manejo de las prótesis de los cuales presentaron anormalidad en los N.F.S.R. 22 (39.3\%) observándose una diferencia estadísticamente significativa $(\mathrm{p}=0.030)$, a diferencia de los N.F.S.E. 15 (33.3\%) donde no se observaron diferencias estadísticamente significativas $(\mathrm{p}=0.526)$ (Tabla 5). En el modelo final de N.F.S.E. sólo permanecía la variable ansiedad asociada a la alteración del flujo salival estimulado que muestra una relación 4 veces mayor de reducción I.C. [2.16;7.39] $(p=0.024)$ entre aquellos que registraron ansiedad comparados con los que no registraban esta condición, el consumo de medicamentos mostró una relación protectora 0.46 I.C. [0.20;1.07] $(p=0.073)$ entre aquellos que consumían medicamentos comparados con los que no consumían, sin embargo, es una relación marginalmente significativa.

En el modelo final de N.F.S.R. sólo se observó asociación de las variables consumo de alimentos sólidos como factor protector de 0.65 I.C. [0.45;0.96] entre las personas que consumían este tipo de alimentos y las que no lo consumían con una diferencia estadísticamente significativa $(p=0.028)$ (Tabla 6$)$.

Tabla 5. Aspectos relacionados con las prótesis dentales y su relación con los niveles flujo salival estimulado y en reposo disminuidos.

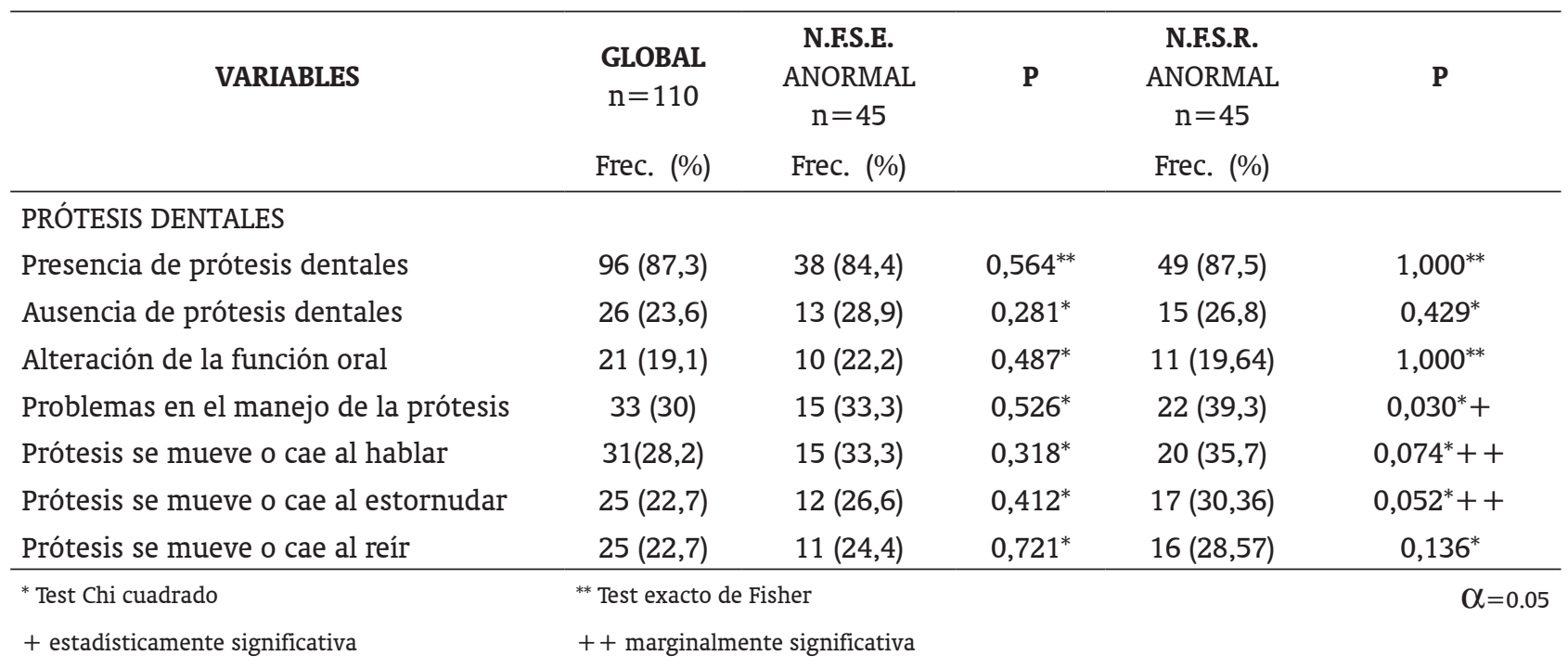


Tabla 6. Modelo de regresión binomial de alteración del flujo salival estimulado. Análisis bivariado.

\begin{tabular}{lccc}
\hline \multicolumn{1}{c}{ VARIABLE } & RR & IC 95\% & P \\
\hline Ansiedad & 2.06 & $0.89 ; 4.74$ & $0.087++$ \\
Hipertensión & 3.31 & $1.89 ; 5.79$ & $<0.001+$ \\
Consumo de medicamentos & 2.55 & $1.51 ; 4.31$ & $<0.001+$ \\
Antihipertensivos & 2.98 & $1.77 ; 5.01$ & $<0.001+$ \\
Hipoglicémicos & 4.51 & $2.80 ; 7.26$ & $<0.001+$ \\
Consumo $\leq 3$ vasos de agua/día & 1.81 & $1.17 ; 2.79$ & $0.007+$ \\
Frecuencia de hacer deporte & 0.63 & $0.41 ; 0.97$ & $0.04+$ \\
Consumir alimentos sólidos & 0.61 & $0.37 ; 1.02$ & $0.063++$ \\
\hline + estadísticamente significativo & ++ marginalmente significativo & $\alpha=0.05$
\end{tabular}

\section{Análisis multivariado (modelo ajustado):}

En el modelo final solo permanecía la variable ansiedad asociada a la alteración del flujo salival estimulado con una relación 4 veces mayor de reducción I.C. [2.16;7.39] $(p=0.024)$ entre aquellos que registraron ansiedad comparados con los que no registraban esta condición, el consumo de medicamentos mostró una relación protectora 0.46 I.C. $[0.20 ; 1.07](p=0.073)$ entre aquellos que consumían medicamentos comparados con los que no consumían, sin embargo es una relación marginalmente significativa (Tabla 7).

\section{Flujo salival en reposo}

\section{Análisis bivariado:}

$\mathrm{Al}$ analizar la razón de prevalencia según factores asociados a la reducción del flujo salival en reposo se encontró que la hipertensión mostró una asociación 2 veces mayor de reducción I.C. $[1.51 ; 3.36](p<0.001)$ entre quienes reportaron tener esta condición y los que no. Con respecto al consumo de medicamentos se encontró una relación 2 veces mayor I.C. [1.34; 3.03] $(p<0.001)$ entre quienes consumían y no consumían medicamentos. Las personas que consumían anti- hipertensivos mostraron una asociación dos veces mayor I.C. [1.51; 3.39] $(\mathrm{p}<0.001)$ entre los que consumían y no consumían este tipo de medicamentos, a diferencia de las que consumían hipoglicemiantes donde se encontraron asociaciones de tres veces mayor I.C $[2.14 ; 4.44](\mathrm{p}<0.001)$ entre quienes consumían y no consumían estos medicamentos. En cuanto a la frecuencia de la práctica de deportes se mostró una relación protectora en cuanto a la disminución del nivel de flujo salival de 0.67 I.C. [0.47; $0.96](p=0.029)$ entre quienes practicaban deporte $y$ los que no lo practicaban (Tabla 8).

\section{Análisis multivariado (modelo ajustado):}

En el modelo final se observó asociación de las variables consumo de alimentos sólidos como factor protector de 0.65 I.C. [0.45;0.96] $(p=0.028)$ entre las personas que consumían este tipo de alimentos y las que no lo consumían, al igual que la variable de otras enfermedades que mostró una relación protectora de 0.32 I.C. [0.10;1.051] $(p=0.061)$ entre las personas que sufrían otras enfermedades y las que sufrían de hipertensión; sin embargo, en esta variable se observó una diferencia marginalmente significativa (Tabla 9).

Tabla 7. Modelo de regresión binomial final ajustado a la alteración de flujo salival estimulado.

\begin{tabular}{lccc}
\hline \multicolumn{1}{c}{ VARIABLE } & RR & IC 95\% & P \\
\hline Ansiedad & 3.99 & $2.16 ; 7.39$ & $0.024+$ \\
Consumo de medicamentos & 0.46 & $0.20 ; 1.07$ & $0.073++$ \\
Consumo Antihipertensivos & 2.65 & $0.53 ; 13.29$ & 0.235 \\
\hline + estadísticamente significativo & & ++ marginalmente significativo & $\alpha=0.05$
\end{tabular}

Tabla 8. Modelo de regresión binomial de alteración de flujo salival en reposo.

\begin{tabular}{lccc}
\hline \multicolumn{1}{c}{ VARIABLE } & RR & IC 95\% & P \\
\hline Hipertensión & 2.25 & $1.51 ; 3.36$ & $<0.001+$ \\
Consumo de medicamento & 2.01 & $1.34 ; 3.03$ & $<0.001+$ \\
Consumo antihipertensivos & 2.27 & $1.51 ; 3.39$ & $<0.001+$ \\
Consumo hipoglicémicos & 3.08 & $2.14 ; 4.44$ & $<0.001+$ \\
Frecuencia de hacer deporte & 0.67 & $0.47 ; 0.96$ & $0.029+$ \\
\hline + estadísticamente significativo & & $\alpha=0.05$ &
\end{tabular}


Tabla 9. Modelo de regresión binomial ajustado de alteración de flujo salival en reposo.

\begin{tabular}{lccc}
\hline \multicolumn{1}{c}{ VARIABLE } & RR & IC 95\% & P \\
\hline Consumo de alimentos sólidos & 0.65 & $0.45 ; 0.96$ & $0.028+$ \\
Otras enfermedades & 0.32 & $0.10 ; 1.051$ & $0.061++$ \\
\hline+ estadísticamente significativo & & ++ marginalmente significativo & $\alpha$
\end{tabular}

\section{DISCUSIÓN}

En este estudio se encontró que la condición sistémica más relacionada en cuanto a la alteración del flujo salival estimulado fue la presencia de ansiedad ya que ésta reportó una relación 4 veces mayor IC $95 \%$, relacionada con la alteración del flujo salival con una significancia estadística $(\mathrm{p}=0.024)$. Con respecto al nivel de flujo salival en reposo la condición que más se relacionó con la disminución de este fue la hipertensión ya que demostró una asociación de 2.25 veces mayor IC 95\% con relación a la disminución del flujo salival una diferencia estadísticamente significativa $(\mathrm{p}<0.001)$.

Otros estudios demostraron que los diuréticos son los medicamentos con más tendencia a producir xerostomía, y que con respecto a la edad los pacientes mayores de 60 años que tomaban antihipertensivos tienen 2 veces más probabilidad de sufrir de xerostomía que aquellos menores de esta edad y en cuanto al género se presentó más en mujeres que consumían antihipertensivos que en hombres. ${ }^{8}$ En este estudio la edad y el género no mostraron evidencia de generación de alteraciones en el flujo salival contrario a lo que afirman otros autores acerca de la asociación de envejecimiento y xerostomía. ${ }^{8,12}$ Aunque en este estudio hubo una asociación marginalmente significativa entre sensación de boca seca (xerostomía) y disminución del fuljo salival estimulado con un $\mathrm{p}=0.052$ y hubo una significancia estadística para el flujo salival reposo $\mathrm{p}=0.022$ en adultos mayores sin tener en cuenta consumo de medicamentos o factores causales de hiposalivación que puedan justificarlo como lo afirma Roisinblit en su revisión. ${ }^{14}$

González y colaboradores reportaron que no hay diferencia estadísticamente significativa entre edad e hiposalivación en una población de 90 hombres y mujeres hipertensos que toman diferentes medicamentos para su tratamiento y un grupo control. ${ }^{9}$

Bergdhal y Bergdahl reportaron sequedad subjetiva relacionada con estrés, ansiedad y depresión. ${ }^{13}$ Otros autores relacionan la diabetes con xerostomía e hiposalivación, provocada por el aumento de la diuresis y la disminución del volumen del líquido extracelular, además de la posible participación de neuropatía diabética, la cual puede producir disminución del tono muscular de labios y carrillos que promueve al desajuste de las prótesis removibles. ${ }^{15-17}$ Por tal razón, esta condición sistémica no fue considerada como criterio de inclusión.

Por otro lado, los medicamentos que han sido relacionados con la hiposalivación han sobrepasado el número de $400 .{ }^{8}$ En este estudio se concentró a partir del consumo de antihipertensivos, ansiolíticos, antiglicémicos, anticonvulsionantes y broncodilatadores. Donde se observó una asociación significativa tanto en flujo salival estimulado $27(60 \%)$ como en reposo 30 (53.5\%) con relación al consumo de medicamentos de tipo antihipertensivos

González y colaboradores reportaron pacientes que presentaban percepción de boca seca de los cuales el $77.77 \%$ consumían antihipertensivos. ${ }^{9}$ En cuanto al tiempo de consumo de medicamentos relacionado con la presencia de xerostomía no se pudo hallar una diferencia estadísticamente significativa ya que un $27.78 \%$ tomaban diuréticos, un $25.93 \%$ ingerían betabloqueadores y $24.08 \%$ con inhibidores de la enzima convertidora de angiotensina. Con una leve tendencia en el sexo femenino y a mayor tiempo de consumo del medicamento menor percepción de xerostomía.

Otros medicamentos como los anticonvulsionantes, antiglicémicos y betabloqueadores no reportaron en este estudio relación con la disminución de los niveles de flujo salival estimulado y en reposo.

La disminución de la masticación reportó presencia de anormalidades de los niveles de flujo salival estimulado con 16 (35.6\%) y un $\mathrm{p}=0.004$ y en reposo $19(33.9 \%)$ con $\mathrm{p}=0.002$, observándose diferencias estadísticamente significativas.

Como lo reportan Yeh y colaboradores quienes explican la relación que existe entre los niveles de flujo salival y la fuerza de mordida, donde se demuestra que entre menor sea la consistencia de la dieta menor va a ser la funcionalidad de las glándulas salivales y, a su vez, menor son los niveles de flujo salival secretados por éstas. ${ }^{18}$ Lo que lleva a deducir que a mayor estimulo masticatorio mayor serán los niveles de flujo salival estimulado. En este estudio se observó que el consumo de alimentos sólidos se presenta como un factor protector para evitar la disminución del flujo salival estimulado RR 0.61 y en reposo RR 0.65 , con una diferencia marginalmente significativa $(p=0.063)$ para flujo salival estimulado y una diferen- 
cia estadísticamente significativa para flujo salival en reposo $(\mathrm{p}=0.028)$.

Otros procesos como la pérdida de líquidos ya sea relacionada con deporte o sudoración excesiva o por otras enfermedades fueron estudiados y se reporta un flujo anormal en reposo en quienes no practicaban deportes, sin estar relacionada al consumo o no de medicamentos o enfermedad sistémica. La sudoración excesiva y práctica de deportes no reportaron datos significativos, por el contrario, en el análisis multivariado se mostró una relación protectora para los niveles de flujo salival estimulado con RR 0.63 I.C. (0.41-0.97) y se observó una diferencia estadísticamente significativa $(p=0.04)$ en cuanto a la práctica deportiva.

Acerca de los pacientes usuarios de prótesis, presentaron problemas con su manejo 33 (30\%) de los cuales, el $22(39,3 \%)$ presentaron anormalidad de los niveles reflujo salival en reposo con una diferencia estadísticamente significativa $(p=0,030)$. Esto es corroborado en la revisión realizada por Roisinblit y colaboradores donde afirma que la falta de lubricación por parte de la saliva en las mucosas genera alteración de las propiedades retentivas de las prótesis. ${ }^{14}$

\section{CONCLUSIONES}

Se pudo determinar que existe una relación directa entre el consumo de medicamentos y la disminución de los niveles de flujo salival estimulado y en reposo.

Los medicamentos que más relación tuvieron con la disminución del flujo salival estimulado fueron los de tipo antihipertensivos y la condición sistémica que mostró ser un factor de riesgo mayor para la disminución del flujo salival estimulado fue la ansiedad.

Con respecto al nivel de flujo salival en reposo los medicamentos que presentaron mayor relación con la disminución del flujo fueron los de tipo antihipertensivos y antihipoglicémicos; además la condición sistémica más asociada a la disminución de los niveles de flujo salival en reposo fue la hipertensión.

Se determinó que el consumo de alimentos sólidos y el consumo de más tres vasos de agua por día contribuyen en gran parte al mejoramiento tanto de los niveles de flujo salival estimulado, como en reposo.

\section{BIBLIOGRAFÍA}

1. Aagaard A, Godiksen S, Teglers P, Schiodt M, Glenert U. Comparison between new saliva stimulants in patients with dry mouth: a placebo-controlled double-blind cross- over study. J Oral Pathol Med 1992; 21: 376 - 380.

2. Aguilar LF, Romero MC. La saliva: revisión sobre composición, función y usos diagnósticos. Primera parte. Univ Odontol 2003; 23:18 - 24. .

3. González M, Montes De Oca L, Jiménez G. Cambios en la composición de la saliva en pacientes gestantes y no gestantes. Perinatol Reprod Hum 2001; 15: 195 - 201.

4. Atkinson JC, Fox PC. Xerostomía. Gerodontics 1986; 2: $193-197$

5. Bayraktar G, Kazancioglu R, Bozfakioglu S, Ecder T, Yildiz A, Ark E. Stimulated salivary flow rate in chronic hemodialysis patients. Nephron 2002; 91: 210 - 214.

6. Ghezzi EM, Lange LA, Ship JA. Determination of variation of stimulated salivary flow rates. J Dent Res 2000; 79: $1874-1878$.

7. Corona Hernández L, Del Rio Navarro B, Fragoso Ríos R, Cuairan Ruidiaz V. Efecto de la terapia con salmeterol y salmeterol con beclometasona inhalados sobre el volumen del flujo salival y la cantidad de IgA secretora en pacientes con asma crónica moderada persistente. División de Estudios de Posgrado e Investigación 2002; 6: 32 - 39 [en línea]. URL disponible en http://www.pve.unam.mx/ alerta/pdf/efecto.pdf

8. Garcia-Pola MJ, Sanmartin P, Letic-Gravilovic A. Xerostomía. FMC 1999; 6: 229 - 236.

9. González CA, Harrah VP, Yepes JF, Percepción de xerostomía en un grupo de pacientes adultos hipertensos. Universitas Odontológica 2002; 22: 15 - 19.

10. Navazesh M, Mulligan R, Komaroff E, Redford M, Greenspan D, Phelan J. The prevalence of xerostomia and salivary gland hypofuntion in a cohort of HIV-positive and at-risk women. J Dent Res 2000; 79: 1502 - 1507.

11. Pinto R. Xerostomía: evaluación y diagnóstico. Facultad de odontología, Universidad Mayor.

12. Thompson WM, Williams SM. Further testing of xerostomia inventory. Oral Surg Oral Med Oral Pathol Oral Radiol Endod 2000; 89: 46 - 50 .

13. Berdahl M, Berdahl J. Low stimulated salivary flow and subjective oral dryness: association with medication, anxiety, depression and stress. J Dent Res 2000; 79: 1652 - 1658.

14. Roisinblit R, Lopez L, Paszucki L, Feldfeber J, Herszkowicz. La "boca seca" en los ancianos. Revista de la Asociación Odontológica Argentina 88: 27 - 33.

15. García J, Boj J, Espasa De Deza E. Influencia del control y de la duración de la diabetes mellitus no insulinodependiente sobre la salud bucodental. ELDR, 20005 (22). Barcelona, España.

16. García-Pola MJ, Vallejo A, Vallejo A, Álvarez I, Lapiedra R. Manifestaciones bucales de la diabetes mellitus. URL disponible en: http://www.coem.org/revista/anterior/0198/ articulo.htm

17. Gay O. Actualidades en el manejo dental del paciente diabético. Revista de la Asociación Dental Mexicana 1999; 56: $18-26$.

18. Yeh C, Johnson D, Doods M, Sakai S, Rugh J, Hatch J. Association of salivary flow rates with maximal bite force. J Dent Res 2000; 79: 1560 - 1566. 